\title{
Systemic lupus or HIV: The wolf also shall dwell with the lamb
}

\author{
Hala I El Gendy* and Gehad G Maghraby \\ Department of Internal Medicine, Rheumatology and Immunology Unit, Cairo University, Cairo, Egypt
}

\begin{abstract}
Background: Systemic lupus erythematosus (SLE) is rarely reported in association with HIV infection. The coincidence of autoimmune diseases in the setting of immunocompetence loss during HIV infection may seem paradoxical.

Case Report: A 35-year-old woman presented with a 9-month history of diffuse progressive colicky abdominal pain, weight loss, and watery diarrhoea. Colonoscopic biopsy revealed nonspecific proctocolitis. Steroids plus mesalazine were initiated with no improvement. Few months later, she suffered from alopecia, arthritis of the small joints, photosensitivity associated with fever and abnormal behaviour in the form of aggression, depressed mood, diminished interest in daily activities as well as a decline in her self-care, memory impairment and deficits in attention. On physical examination patient was pale, emaciated, malar rash and oral candidiasis were noted higher cerebral functions examination revealed impaired sustained attention recent memory and restricted affect. Neurological examination revealed with bilateral upper limb tremors, paraparesis symmetrical rigidity, and exaggerated deep tendon reflexes. Plantar reflexes were bilaterally extensor.

Blood tests revealed pancytopenia, with reticulocytosis, positive coomb's test and ESR $100 \mathrm{~mm} / \mathrm{h}$. ANA test was positive homogenous with titre $1 / 320$. Anti-ds DNA was negative and Hepatic and renal parameters were normal. HIV by ELISA and Western Blot was positive. Her CD4 count was $44 / \mathrm{mm}^{3}$. Viral load by PCR was HIV RNA 68,962, other virology screen was negative copies/ml). Magnetic resonance imaging of the brain revealed diffuse cortical atrophy, thick sheets of abnormal MR signals were seen within the paraventricular white matter and subcortical level, with capacious ventricular system.
\end{abstract}

Conclusion: HIV could be a great mimicker and we suggest screening for HIV more often than is generally done now in the context of evaluation of suspected autoimmune disease.

\section{Introduction}

Human immunodeficiency virus (HIV) infection and systemic lupus erythematosus (SLE) have a large number of clinical features in common (fever, lymphadenopathy, rash, renal, neurologic, and hematologic involvement, etc.). Differentiating the 2 diseases has been difficult at times since weak positive antinuclear antibodies (ANA) have been found in patients with HIV, and false-positive HIV test results in patients with SLE [1].

It has been recently suggested that the increased production of autoantibodies in SLE patients could be the factor saving from the development of AIDS. However, a rare coexistence of HIV infection and SLE can occur. Raising the awareness of physicians dealing with HIVinfected patients of this possible correlation appears to be significant issue, because of the fact that both clinical and laboratory findings in HIV infection and SLE are similar.

\section{Case Report}

A 35-year-old married woman gave a history of diffuse colicky abdominal pain of 9 months duration with progressive. It was associated with watery diarrhoea (about 4 motions per day), with no blood or mucous. Progressive weight loss was also noted. Colonoscopy and biopsy revealed nonspecific proctocolitis. Steroids (prednisone tablets $30 \mathrm{mg} /$ day) plus mesalazine tablets ( $2 \mathrm{~g} /$ day $)$ were initiated however pain and diarrhoea did not improved.

Six months later, she suffered from alopecia, arthritis of the small joints, photosensitivity associated with fever $\left(38^{\circ} \mathrm{C}\right.$ all day $) .400 \mathrm{mg} /$ day Hydroxychloroquine was added by her treating physician.
Four months ago, the patient reported symptoms of abnormal behaviour in the form of aggression, depressed mood, diminished interest in daily activities as well as a decline in her self-care. The patient showed increasing forgetfulness and memory impairment and deficits in attention. She displayed hand tremors and inability to walk in the past few weeks (paresis).

On examination, she appeared emaciated and pale, with malar rash and oral candidiasis. The patient was conscious, with impaired sustained attention and recent memory. She scored 19 of 30 on the Mini-Mental Status Examination.

She had restricted affect with bilateral upper limb action tremors, symmetrical rigidity, and exaggerated deep tendon reflexes. Plantar reflexes were bilaterally extensor.

We investigated the patient to rule out an underlying connective tissue disorder. Her ANA screen was positive homogenous, 1/320 titre, negative anti-double stranded DNA\& normal C3\&C4. Other significant findings included an elevated erythrocyte sedimentation rate (ESR $100 \mathrm{~mm} / \mathrm{h}$ hour) and pancytopenia, with evidence suggestive of a haemolytic anaemia with reticulocytosis, positive coomb's test.

${ }^{\star}$ Correspondence to: Hala Ibrahem El Gendy, Internal Medicine department, Kasr Alainy School of Medicine, Cairo University, Cairo, Egypt, E-mail: hei12001@yahoo.com

Received: January 10, 2019; Accepted: January 15, 2019; Published: January 21 2019 
Her hepatic and renal parameters were within normal limits. Virology screen was negative. HIV by ELISA and Western Blot was positive. Her CD4 count was $44 / \mathrm{mm}^{3}$, viral load by PCR was HIV RNA 68,962 copies $/ \mathrm{ml}$.

Magnetic resonance imaging of the brain revealed diffuse cortical atrophy (Figures 1 and 2), thick sheets of abnormal MR signals were seen within the paraventricular white matter reaching the subcortical level. Capacious ventricular system and prominent cortical sulci, Sylvian fissure and basal cisterns were also noted.

The CSF analysis revealed clear, cellular fluid with high normal protein level and normal glucose. It was negative for acid-fast bacilli by Ziehl-Neelsen stain.

\section{Discussion}

The association between autoimmune diseases and retroviral infections has been studied extensively. It is well documented that the presentation of HIV infection can mimic that of autoimmune phenomena. Fever, lymphadenopathy, rash, renal dysfunction, neurological and haematological disorders, sicca syndrome and polyarthralgias have been described [2].

Photosensitivity is a frequently reported dermatological manifestation of HIV infection and is generally considered to be a manifestation of late HIV infection [3].

Opportunistic infections like candidiasis have also been reported in SLE [4].

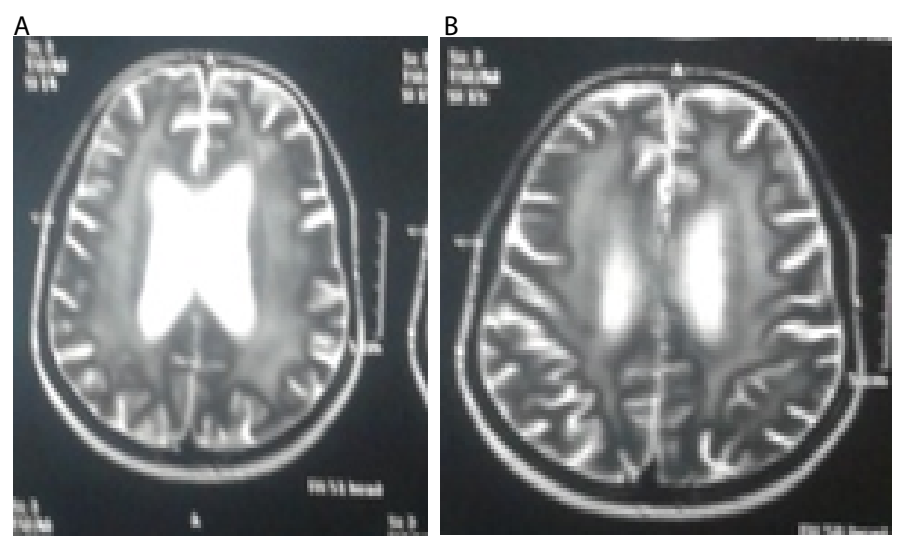

Figure 1. Diffuse cortical atrophy thick sheets of abnormal MR signals were seen within the paraventricular white matter reaching the subcortical level

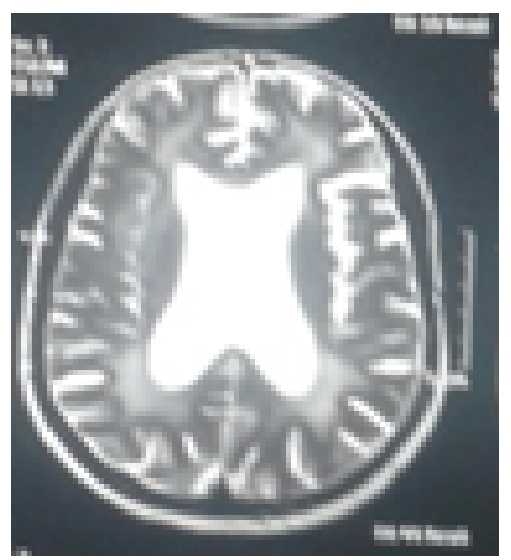

Figure 2. Capacious ventricular system and prominent cortical sulci, Sylvian fissure and basal cisterns were also noted
HIV infection may induce production of autoantibodies due to the structural antigen similarity between viral proteins and self-antigens. The molecular mimicry between HIV protein and self-antigens could cause antibody cross-reactions and lead to development of autoimmune disease [5].

CD4 T-cells and dendritic cells play an important role in the pathogenesis of systemic lupus and they are depleted in HIV infection. There are recorded cases of SLE in patient with CD4 count of 0 cells/ $\mu \mathrm{L}$ with improvement of rheumatic manifestations after antiviral therapy suggesting that CD4 cells and plasmacytoid dendritic cells (pDCs) are not the only factors in this immunological disease [6].

It has been recently suggested that the presence of autoimmune diseases like SLE may in some way be protective for HIV through production of self-reactive antibodies that recognize and neutralize HIV-1 [7].

In our patient, virtually all her symptoms would have been consistent with both SLE and HIV infection. Photosensitivity, loss of weight, alopecia arthritis and fatigue are well-known components of lupus, as are the findings of a strongly positive ANA and pancytopenia. The patient met five out of the eleven symptoms of the revised 2015 ACR/SLICC criteria for diagnosis of SLE, namely photosensitivity, alopecia, haematological disorder, joint disease and antinuclear antibody. However, as we have discussed, all of the above can be explained on the basis of an underlying HIV infection. In our patient, the rapid progressive cognitive and behavioral impairment that was seen along with prominent extra pyramidal and pyramidal features with MRI findings could not be explained by systemic lupus, HIV associated dementia is typically rapidly progressive with a mean survival of about 6 months,with rapid course in those with CD4 counts of $<100$ progress more rapidly [8]. Our patient CD4count was $44 / \mathrm{mm}^{3}$.

Also in our patient hydroxychloroquine was initiated with no improvement although, Piconi et al. [9], demonstrated its ability to inhibit the post transcriptional production of HIV-1 in monocytes and T cells so it could have a protective role of against HIV.

We would like to stress on the importance of doing a serological screening to rule out HIV infection in cases of suspected autoimmune disease. HIV has by now probably replaced syphilis as the "great mimicker" and we suggest screening for HIV more often than is generally done now in the context of evaluation of suspected autoimmune disease. Similarly, it should be kept in mind that photosensitivity is a definite, well-documented feature of HIV infection and may be the initial manifestation of HIV disease. It typically occurs in patients who have a CD4 T lymphocyte count $\leq 50$ cells/ $\mu \mathrm{l}$ and worsens as the immunodeficiency progresses [10].

Finally, the question of the validity of the diagnostic criteria of lupus in the setting of HIV positivity would probably requires further refinement in the future.

\section{References}

1. Jian L, Liang W, Zhang Y, Li L, Mei Y, et al. (2015) Systemic lupus erythematosus patient with false positive results of antibody to HIV: A case report and a comprehensive literature review. Technol Health Care 23: S99-S103. [Crossref]

2. Calabrese LH, Kirchner E, Shrestha R (2005) Rheumatic complications of human immunodeficiency virus infection in the era of highly active antiretroviral therapy: emergence of a new syndrome of immune reconstitution and changing patterns of disease. Semin Arthritis Rheum 35:166-174. [Crossref] 
3. Vin-Christian K, Epstein JH, Maurer TA, McCalmont TH, Berger TJ (2000) Photosensitivity in HIV-infected individuals. J Dermatol 27: 361-9. [Crossref]

4. Ginzler EM (2002) Infections in systemic lupus erythematosus. In: Wallace DJ, Hahn BH (Eds.), Dubois' lupus erythematosus. (6thedn), Philadelphia: Lippincot Williams and Wilkins; 917-26.

5. Carugati M, Franzetti M, Torre A, Giorgi R, Genderini A, et al. (2013) Systemic lupus erythematosus and HIV infection: a whimsical relationship. Reports of two cases and review of the literature. Clin Rheumatol 32: 1399-405. [Crossref]

6. Gould T, Tikly M (2004) Systemic lupus erythematosus in a patient with human immunodeficiency virus infection-challenges in diagnosis and management. Clin Rheumatol 23:166-169. [Crossref]
7. Bonsignori M, Wiehe K, Grimm SK, Lynch R, Yang G, et al. (2014) An autoreactive antibody from an SLE/HIV-1 individual broadly neutralizes HIV-1. J Clin Invest 124 1835-43. [Crossref]

8. Dore GJ, McDonald A, Li Y, Kaldor JM, Brew BJ (2003) Marked improvement in survival following AIDS dementia complex in the era of highly active antiretroviral therapy. AIDS 17: 1539-1545. [Crossref]

9. Piconi S, Parisotto S, Rizzardini G, et al. (2011) Hydroxychloroquine drastically reduces immune activation in HIV-infected, antiretroviral therapy-treated immunologic non-responders. Blood 118: 3263-3272. [Crossref]

10. Bilu D, Mamelak AJ, Nguyen RH, Queiroz PC, Kowalski J (2004) Clinical and epidemiologic characterization of photosensitivity in HIVpositive individuals. Photodermatol Photoimmunol Photomed 20: 175-183. [Crossref]

Copyright: @2019 El Gendy HI. This is an open-access article distributed under the terms of the Creative Commons Attribution License, which permits unrestricted use, distribution, and reproduction in any medium, provided the original author and source are credited. 